\title{
COMMENTARY
}

\section{Contrast-enhanced ultrasound: a new vision of microcirculation in the intensive care unit}

\author{
Anatole Harrois and Jacques Duranteau ${ }^{*}$ \\ See related research by Schneider et al., http://ccforum.com/content/17/4/R138
}

\begin{abstract}
To gain new insights into renal perfusion and pathogenesis of acute kidney injury in intensive care unit (ICU) patients, we need new techniques to evaluate renal microcirculation. In addition, a bedside technique applicable in the ICU could be extremely useful for physicians to adjust the optimal therapeutic/preventive modalities for kidney perfusion in each patient. Contrast-enhanced ultrasound (CEUS) has been validated to assess and quantify the microcirculation up to capillary perfusion in several organs. In a recent issue, Schneider and colleagues suggest that CEUS is feasible, well tolerated and able to quantify cortical renal microcirculation in patients undergoing cardiac surgery. In addition, CEUS derived-parameters suggest a decrease in renal perfusion occurring within 24 hours of surgery in patients at risk of acute kidney injury. This study opens up new possibilities for the assessment of cortical renal microcirculation in ICU patients.
\end{abstract}

Despite our increasing ability to support vital organs and resuscitate patients, acute kidney injury (AKI) is a frequent complication of cardiac surgery and increases morbidity and mortality. The primary resuscitation strategy for these patients is to restore effective renal blood flow (RBF) by fluid resuscitation to maintain adequate renal microcirculation and parenchymal oxygen and thereby prevent AKI. However, renal hypoperfusion may persist despite restoration of global hemodynamics due to inadequate RBF and renal microcirculatory dysfunction. Thus, defining the adequacy of resuscitation requires attention to both global and regional perfusion. In addition, renal hypoperfusion is not the only cause for

* Correspondence: jacques.duranteau@bct.aphp.fr

AP-HP, Service d'Anesthésie-Réanimation, Hôpitaux Universitaires Paris-Sud, Université Paris-Sud, Hôpital de Bicêtre, Le Kremlin-Bicêtre 94275, France development of $\mathrm{AKI}$, and the role of RBF alterations in the pathogenesis of AKI is still debated. To gain new insights into renal perfusion and pathogenesis of AKI in intensive care unit (ICU) patients, we need new techniques to evaluate RBF and kidney microcirculation. In addition, a bedside technique applicable in the ICU could be extremely useful for physicians to adjust the optimal therapeutic/preventive modalities for kidney perfusion in each patient.

In this context, a study in this issue of Critical Care provides useful important new information. Schneider and colleagues [1] reported for the first time that contrastenhanced ultrasound (CEUS), a recent technique for quantification of tissue perfusion and microcirculation, is able to assess renal cortical perfusion in ICU patients before and after cardiac surgery. Schneider and colleagues conclude that CEUS appears feasible and well tolerated. In addition, CEUS derived-parameters suggest a decrease in renal perfusion occurring within 24 hours of surgery in patients at risk of AKI.

CEUS uses microbubble contrast agents, which are composed of microbubbles of an injectable gas in a supporting shell (phospholipids or proteins) [2-4]. Their size (1 to $6 \mu \mathrm{m})$, similar to red blood cells, allows microbubbles to cross the capillary bed of the pulmonary circulation and to flow up to capillary level of the other organs, enabling assessment of their microcirculation. At the same time, their size is big enough that they do not cross the endothelium, making them true intravascular agents. Ultrasound waves induce a nonlinear oscillation of microbubbles and the backscattered signal consequently contains a range of frequencies in addition to that of the incident ultrasound field with a high echogenicity difference between the gas in the microbubbles and the soft tissue. The microbubbles can be injected as a bolus injection or as a constant infusion. When a constant infusion is administered, as in the study of Schneider and colleagues [1], a 'destruction-replenishment' mode is applied in which higher power ultrasound pulses are used to destroy 
the bubbles followed by low-power ultrasound pulses to observe replenishment in the tissue. Signal-processing techniques have been developed to separate echoes generated by microbubbles from those generated by tissue.

CEUS has been validated to assess tissue perfusion in several organs. For example, CEUS has a high diagnostic performance in the detection and characterization of liver and renal tumors [5-7]. It allows the quantification of myocardial blood flow in humans and provides the basis for the noninvasive and quantitative assessment of coronary artery disease [8]. Endoleaks after endovascular aortic repair could be time- and cost-effectively detected by CEUS [9]. In addition, CEUS demonstrates improved diagnostic value compared to Doppler ultrasonography for the detection of chronic allograft nephropathy after renal transplantation [10].

Significant variations in quantitative CEUS results have been described, however [3]. Several factors may induce significant variability in quantitative CEUS - scanner settings, patient factors (blood pressure, tissue motion), and bubble types and handling. Thus, it is crucial to demonstrate that CEUS could be an effective technique and a clinically useful tool in ICU patients to assess organ perfusion and the impact of our therapeutic strategies.

The study of Schneider and colleagues [1] is a first step towards demonstrating the feasibility of CEUS to assess cortical renal microcirculation in ICU patients. Schneider and colleagues [1] demonstrated that this technique is safe in ICU patients without deleterious hemodynamic effects. Despite the fact that some patients were hemodynamically unstable with vasopressor infusion, the authors were able to obtain adequate contrast enhancement for all patients at all time points. Finally, the authors found an excellent correlation between readings for the perfusion index and the relative blood volume. This last result highly suggests that the analysis of video sequences is reproducible. However, there is clearly important variability between basal values and within each patient between each study time-point. This might be due to individual differences and heterogeneities in cortical microcirculation but could also be related to acquisition issues like scanner settings, placement of the region of interest, and so on. Thus, it is essential to take into account the variability of CEUSderived parameters between measurements in the same patient at the same time-point. This point is critical to confirm that the observed differences are higher than potential differences due to the variability of acquisition of CEUS-derived parameters.

The study of Schneider and colleagues opens up new possibilities for the assessment of cortical renal microcirculation in ICU patients. Further studies are required to confirm these results in a higher number of patients. Being able to quantify renal microcirculation is the first step toward developing interventions that target microcirculatory perfusion and prevent AKI.

\section{Abbreviations}

AKI: Acute kidney injury; CEUS: Contrast-enhanced ultrasound; ICU: Intensive care unit; RBF: Renal blood flow.

\section{Competing interests}

The authors declare that they have no competing interests.

\section{Published: 23 August 2013}

\section{References}

1. Schneider AG, Goodwin MD, Schelleman A, Bailey M, Johnson L, Bellomo R: Contrast-enhanced ultrasound to evaluate changes in renal cortical perfusion around cardiac surgery: a pilot study. Crit Care 2013, 17:R138.

2. Schneider A, Johnson L, Goodwin M, Schelleman A, Bellomo R: Bench-tobedside review: contrast enhanced ultrasonography - a promising technique to assess renal perfusion in the ICU. Crit Care 2011, 15:157.

3. Tang MX, Mulvana H, Gauthier T, Lim AKP, Cosgrove DO, Eckersley RJ, Stride E: Quantitative contrast-enhanced ultrasound imaging: a review of sources of variability. Interface Focus 2011, 1:520-539.

4. Le Dorze M, Bougle A, Deruddre S, Duranteau J: Renal Doppler ultrasound. Shock 2012, 37:360-365.

5. Larsen LPS: Role of contrast enhanced ultrasonography in the assessment of hepatic metastases: a review. World J Hepatol 2010, 2:8-15.

6. Helck A, D'Anastasi M, Notohamiprodjo M, Thieme S, Reiser M, Clevert DA: Improved visualization of renal lesions using three-dimensional ultrasound - a feasibility study. Clin Hemorheol Microcirc 2011, 49:537-550.

7. Clevert DA, D'Anastasi M, Jung EM: Contrast-enhanced ultrasound and microcirculation: efficiency through dynamics - current developments. Clin Hemorheol Microcirc 2013, 53:171-186.

8. Vogel R, Indermühle A, Reinhardt J, Meier P, Siegrist PT, Namdar M, Kaufmann PA, Seiler C: The quantification of absolute myocardial perfusion in humans by contrast echocardiography. J Am College Cardiol 2005, 45:754-762.

9. Clevert DA, Minaifar N, Weckbach S, Kopp R, Meimarakis G, Clevert DA, Reiser M: Color duplex ultrasound and contrast-enhanced ultrasound in comparison to MS-CT in the detection of endoleak following endovascular aneurysm repair. Clin Hemorheol Microcirc 2008, 39:121-132.

10. Schwenger V, Korosoglou G, Hinkel UP, Morath C, Hansen A, Sommerer C, Dikow R, Hardt S, Schmidt J, Kücherer H, Katus HA, Zeier M: Real-time contrast-enhanced sonography of renal transplant recipients predicts chronic allograft nephropathy. Am J Transplant 2006, 6:609-615.

doi:10.1186/1364-8535-17-449

Cite this article as: Harrois and Duranteau: Contrast-enhanced ultrasound: a new vision of microcirculation in the intensive care unit. Critical Care 2013 17:449. 